\title{
Renal Artery Stenosis
}

National Cancer Institute

\section{Source}

National Cancer Institute. Renal Artery Stenosis. NCI Thesaurus. Code C123221.

Narrowing of a main artery in the kidney. 